\title{
LOCAL DERIVATIONS OF NEST ALGEBRAS
}

\author{
HAN DEGUANG AND WEI SHUYUN
}

(Communicated by Palle E. T. Jorgensen)

\begin{abstract}
Let $X$ be an arbitrary reflexive Banach space, and let $\mathscr{N}$ be a nest on $X$. Denote by $\mathscr{D}(\mathscr{N})$ the set of all derivations from $\operatorname{Alg} \mathscr{N}$ into $\operatorname{Alg} \mathscr{N}$. For $N \subset \mathcal{N}$, we set $N_{-}=\bigvee\{M \in \mathscr{N}: M \subset N\}$. We also write $0_{-}=0$. Finally, for $E, F \in \mathscr{N}$ define $(E, F]=\{K \in \mathscr{N}: E \subset K \subseteq F\}$. In this paper we prove that a sufficient condition for $\mathscr{D}(\mathscr{N})$ to be (topologically) algebraically reflexive is that for all $0 \neq E \in \mathscr{N}$ and for all $X \neq F \in \mathscr{N}$, there exist $M \in(0, E]$ and $N \in(F, X]$, such that $M_{-} \subset M$ and $N_{-} \subset N$. In particular, we prove that this condition automatically holds for nests acting on finite-dimensional Banach spaces.
\end{abstract}

\section{INTRODUCTION}

In this paper we study local derivations of nest algebras on Banach spaces. Before we define local derivations, we first stipulate that all Banach spaces and Banach algebras considered in this paper will be over the complex number field. Let $\mathscr{A}$ be a Banach algebra. We denote by $\mathscr{D}(\mathscr{A})$ the set of all derivations on $\mathscr{A}$. We say that a linear mapping $\phi: \mathscr{A} \rightarrow \mathscr{A}$ is a local derivation of $\mathscr{A}$ if for all $A \in \mathscr{A}$, there exists a $\delta \in \mathscr{D}(\mathscr{A})$, depending on $A$, such that $\phi(A)=\delta(A)$. The following two questions seem natural: (a) Is every local derivation of $\mathscr{A}$ a derivation? (b) If every operator in $\mathscr{D}(\mathscr{A})$ is norm-continuous, then is every norm-continuous local derivation of $\mathscr{A}$ a derivation? In general, the answer to these questions is negative (see [K]). However, for some special Banach algebras, e.g., von Neumann algebras, the answer is positive; the answer is also positive for $B(X)$, the algebra of all bounded linear operators on a Banach space $X[\mathrm{~K}$, LS]. We find it of interest to study those Banach algebras for which the answer to the above questions is positive.

The above two questions can be reformulated in terms of the notions of algebraic reflexivity and (topological) algebraic reflexivity. These concepts of reflexivity have appeared in various contexts [DH, L, ALVS]. To define these notions of reflexivity, we first establish some notation. Let $V$ be a Banach space. Let $L(V)$ be the algebra of all linear operators on $V$, and let $B(V)$

Received by the editors January 20, 1993 and, in revised form, August 4, 1993, September 7, 1993 and March 24, 1994.

1991 Mathematics Subject Classification. Primary 47D25, 47D30; Secondary 46L40.

Key words and phrases. Local derivation, nest algebras.

Research partially supported by an NSF (of China) grant. 
be the algebra of all bounded linear operators on $V$. If $\mathscr{B} \subseteq L(V)$, we write $\operatorname{ref}_{\mathrm{a}}(\mathscr{B})=\{T \in L(V): T v \in \mathscr{B} v, v \in V\}$ and $\operatorname{ref}_{\mathrm{at}}(\mathscr{B})=\{T \in B(V): T v \in$ $\mathscr{B} v, v \in V\}$. Here, $\mathscr{B} v=\{S v: S \in \mathscr{B}\}$. For $\mathscr{B} \subseteq L(V), \mathscr{B}$ is said to be algebraically reflexive if $\mathscr{B}=\operatorname{ref}_{\mathrm{a}}(\mathscr{B})$; whereas for $\mathscr{B} \subseteq B(V), \mathscr{B}$ is said to be (topologically) algebraically reflexive if $\mathscr{B}=\operatorname{ref}_{\mathrm{ta}}(\mathscr{B})$. If $\mathscr{B} \subseteq L(V)$ and $T \in L(V)$, we say that $T$ is locally in $\mathscr{B}$ if for all $v \in V$ there exists an $S \in \mathscr{B}$ such that $T v=S v$. Using these definitions, observe that for $\mathscr{B} \subseteq L(V), \mathscr{B}$ is algebraically reflexive if and only if for all $T \in L(V), T \in \mathscr{B}$ whenever $T$ is locally in $\mathscr{B}$. Similarly, observe that for $\mathscr{B} \subseteq B(V), \mathscr{B}$ is (topologically) algebraically reflexive if and only if for all $T \in B(V), T \in \mathscr{B}$ whenever $T$ is locally in $\mathscr{B}$. Now let $\mathscr{A}$ be a Banach algebra. In the above definitions, set $V=\mathscr{A}$ and $\mathscr{B}=\mathscr{D}(\mathscr{A})$. Note that for $\phi \in L(\mathscr{A}), \phi$ is in $\mathscr{D}(\mathscr{A})$ if and only if $\phi$ is a local derivation of $\mathscr{A}$. Therefore, according to the observations immediately above, questions (a) and (b) can be reformulated, respectively, as follows: $\left(\mathrm{a}^{\prime}\right)$ Is $\mathscr{D}(\mathscr{A})$ algebraically reflexive? $\left(\mathrm{b}^{\prime}\right)$ If $\mathscr{D}(\mathscr{A}) \subseteq B(\mathscr{A})$, then is $\mathscr{D}(\mathscr{A})$ (topologically) algebraically reflexive? Thus, the aforementioned results of [K] and [LS] can be restated as saying that if $R$ is a von Neumann algebra and $X$ is a Banach space, then $\mathscr{D}(R)$ is (topologically) algebraically reflexive and $\mathscr{D}(B(X))$ is algebraically reflexive.

The main theorem of this paper is Theorem 3.7. To state this theorem, let $X$ be an arbitrary reflexive Banach space, and let $\mathscr{N}$ be a nest on $X$. For $N \subset \mathscr{N}$, let $N_{-}=\bigvee\{M \in \mathscr{N}: M \subset N\}$. We also write $0_{-}=0$. If $E, F \in \mathscr{N}$ define $(E, F]=\{K \in \mathscr{N}: E \subset K \subseteq F\}$. Finally, let $\operatorname{Alg} \mathscr{N}$ be the algebra of all $T \in B(X)$ such that $T(M) \subseteq M$ for all $M \in \mathscr{N}$, and write $\mathscr{D}(\mathscr{N})=\mathscr{D}(\operatorname{Alg} \mathscr{N})$. Then Theorem 3.7 states that every norm-continuous local derivation of $\operatorname{Alg} \mathscr{N}$ is a derivation if the nest $\mathscr{N}$ satisfies the following condition: For all $0 \subset M \in \mathscr{N}$ and for all $X \supset N \in \mathcal{N}$, there exist $E_{1} \in$ $(0, M]$ and $E_{2} \in(N, X]$, such that $\left(E_{1}\right)_{-} \subset E_{1}$ and $\left(E_{2}\right)_{-} \subset E_{2}$. Now, a result obtained in [H1] states that $\mathscr{D}(\mathscr{N}) \subseteq B(\mathrm{Alg} \mathscr{N})$; hence, combining this result with Theorem 3.7, we see that if $\mathscr{N}$ satisfies the last condition, above, then $\mathscr{D}(\mathscr{N})$ is (topologically) algebraically reflexive. We conjecture that this is true even when $X$ and $\mathscr{N}$ are completely arbitrary.

\section{Preliminaries}

Throughout the rest of this paper, $X$ will denote a Banach space and $B(X)$ will denote the algebra of all bounded linear operators on $X$. Moreover, we will assume that all subspaces of Banach spaces are closed and that all operators on Banach spaces are bounded. The symbol $\subset$ will denote proper set inclusion. A nest $\mathcal{N}$ in $X$ is a totally ordered lattice of subspaces of $X$ that contains $0, X$ and is closed under arbitrary closed linear spans, denoted by $\mathrm{V}$, and arbitrary closed intersections, denoted by $\Lambda$. If $N \in \mathscr{N}$ and $N \subset X$, then we write $N_{+}=\bigwedge\{M \in \mathscr{N}: N \subset M\}$. We also write $X_{+}=X$. For a subspace $M$ of $X$, define $M^{\perp}=\left\{f \in X^{*}: f(M)=0\right\}$, where $X^{*}$ is the dual of $X$. If $x \in X$ and $f \in X^{*}$, the rank-one operator $u \mapsto f(u) x$ is denoted by $x \otimes f$. If $M$ is a subspace of $X$ and $T \in B(X)$, then the restriction of $T$ to $M$ is denoted by $\left.T\right|_{M}$.

Lemma 2.1 [Lam]. If $\mathscr{L}$ is a subspace lattice, then $x \otimes f \in \operatorname{Alg} \mathscr{L}$ if and only if there exists an element $L \in \mathscr{L}$ such that $x \in L$ and $f \in\left(L_{-}\right)^{\perp}$. 
Lemma $2.2[\mathrm{H} 3$, Spa]. Let Alg $\mathcal{N}$ be a nest algebra on Banach space $X$. Then the subalgebra of $\operatorname{Alg} \mathscr{N}$ generated by all rank-one operators in $\operatorname{Alg} \mathscr{N}$ is dense in $\operatorname{Alg} \mathscr{N}$ in the strong operator topology.

Let $\mathscr{A}$ be an algebra, and let $\mathscr{M}$ be a bimodule of $\mathscr{A}$. Let $\delta$ be a linear mapping from $\mathscr{A}$ into $\mathscr{M}$. Then $\delta$ is said to be a derivation from $\mathscr{A}$ into $\mathscr{M}$ if $\delta(A B)=A \delta(B)+\delta(A) B$ for all $A, B \in \mathscr{A} . \delta$ is an inner derivation if there exists an element $M \in \mathscr{M}$ such that for all $A \in \mathscr{A}, \delta(A)=M A-A M$. We say $\delta$ is a local derivation if for each $A \in \mathscr{A}$, there exists a derivation $\gamma_{A}: \mathscr{A} \rightarrow \mathscr{M}$, depending on $A$, such that $\delta(A)=\gamma_{A}(A)$.

Recall that a complete lattice $\mathscr{L}$ is said to be completely distributive if the following identity and its dual hold:

$$
\bigwedge_{\alpha \in A} \bigvee_{\beta \in B} L_{\alpha, \beta}=\bigvee_{f \in B^{A}} \bigwedge_{\alpha \in A}\left(L_{\alpha f(\alpha)}\right)
$$

Here, $\left\{L_{\alpha, \beta}: \alpha \in A, \beta \in B\right\}$ is an arbitrary family of elements from $\mathscr{L}$. (See [Lam].)

Lemma 2.3 [H1]. Let $\mathscr{L}$ be a completely distributive subspace lattice of $X$. Then every derivation from $\operatorname{Alg} \mathscr{L}$ into $B(X)$ is continuous.

Note that a nest $\mathscr{N}$ is a completely distributive subspace lattice; therefore, by Lemma 2.3, $\mathscr{D}(\mathscr{N}) \subseteq B(X)$. Combining this with the main result of [H4], we have the following lemma.

Lemma 2.4. Let $\mathscr{N}$ be a nest on a Banach space $X$. Then every derivation from $\operatorname{Alg} \mathscr{N}$ into $\operatorname{Alg} \mathscr{N}$ is inner.

Remark. Corollary 3.8 of [H2] states that if $\mathscr{L}$ is a completely distributive subspace lattice on $X$ and if the first Hochschild cohomology space

$$
H^{1}(\operatorname{Alg} \mathscr{L}, B(X))=0,
$$

then $H^{1}(\operatorname{Alg} \mathscr{L}, \mathscr{M})=0$ for any reflexive bimodule $\mathscr{M}$ of $\mathscr{A}$ that contains $\mathscr{A}$. Then, by Lemma 2.4, we conclude that every derivation from $\operatorname{Alg} \mathscr{N}$ into itself is inner. This will be used in this paper.

\section{LOCAL DERIVATIONS}

Let $\mathscr{N}$ be a nest in a Banach space $X$, and let $\phi$ be a local derivation from $\operatorname{Alg} \mathscr{N}$ into itself. By the remark following Lemma 2.4, $\phi$ has the property that for each $T \in \operatorname{Alg} \mathscr{N}$ there exists $A_{T} \in \operatorname{Alg} \mathscr{N}$ such that $\phi(T)=A_{T} T-T A_{T}$. In the following statements, if we do not stipulate other conditions, the symbols $\mathscr{N}$ and $\phi$ will have the meaning just stated.

Theorem 3.1. Let $N \in \mathscr{N}$ and $N_{-} \neq X$. Then there exist linear mappings $A_{N}: N \rightarrow X$ and $B_{N}^{*}:\left(N_{-}\right)^{\perp} \rightarrow X^{*}$ such that $\phi(x \otimes f)=A_{N} x \otimes f+x \otimes B_{N}^{*} f$ for all $x \in N, f \in\left(N_{-}\right)^{\perp}$.

The proof of this theorem is similar to the proof of related results found in [LS]; therefore, we omit the proof.

Lemma 3.2. Let $\mathbf{C}$ be the field of complex numbers. Then for any $N, M \in \mathscr{N}$ such that $N \subset M$ and $M_{-} \neq X$ there exists $\lambda_{M N} \in \mathbf{C}$ such that $\left.A_{M}\right|_{N}=$ $A_{N}+\lambda_{M N} I_{N}$ and $\left.B_{N}^{*}\right|_{\left(M_{-}\right)^{\perp}}=B_{M}^{*}+\lambda_{M N} I_{\left(M_{-}\right)^{\perp}}$. 
Proof. Fix $f \in\left(M_{-}\right)^{\perp}$ such that $f \neq 0$. Then

$$
\phi(x \otimes f)=A_{N} x \otimes f+x \otimes B_{N}^{*} f, \quad x \in N,
$$

and

$$
\phi(x \otimes f)=A_{M} x \otimes f+x \otimes B_{M}^{*} f, \quad x \in M .
$$

Thus $\left(A_{M}-A_{N}\right) x \otimes f=x \otimes\left(B_{N}^{*}-B_{M}^{*}\right) f$ for all $x \in N$. This implies that for any $x \in N$ there exists $\lambda_{x} \in \mathbf{C}$ such that $\left(A_{M}-A_{N}\right) x=\lambda_{x}$. Because $\left(A_{M}-A_{N}\right)$ is linear on $N$, we have that there exists $\lambda_{M N} \in \mathbf{C}$ such that $\left(A_{M}-A_{N}\right) x=\lambda_{M N}$ on $N$. Here we also have $\left.B_{N}^{*}\right|_{\left(M_{-}\right)^{\perp}}=B_{M}^{*}+\lambda_{M N} I_{\left(M_{-}\right)^{\perp}}$.

Lemma 3.3. There exist a linear mapping $A: \bigcup\left\{N: N \in \mathscr{N}, \mathbf{N}_{-} \neq X\right\} \rightarrow X$ and a linear mapping $B^{*}: \bigcup\left\{\left(N_{-}\right)^{\perp}: N \in \mathscr{N}, N \neq 0\right\} \rightarrow X^{*}$ such that $\phi(x \otimes f)=A x \otimes f+x \otimes B^{*} f$ for all $x \otimes f$ in $\operatorname{Alg} \mathscr{N}$.

Proof. Fix $N \in \mathcal{N}$ such that $N \neq 0$ and $N_{-} \neq X$. Also, fix $M \in \mathscr{N}$ such that $M \neq 0$ and $M_{-} \neq X$. Define $A_{M}^{\prime}$ and $\left(B_{M}^{*}\right)^{\prime}$ as follows:

$$
A_{M}^{\prime}=\left\{\begin{array}{ll}
A_{M}, & \text { if } M=N, \\
A_{M}-\lambda_{M N}, & \text { if } M \supset N, \\
A_{M}+\lambda_{M N}, & \text { if } M \subset N,
\end{array} \quad\left(B_{M}^{*}\right)^{\prime}= \begin{cases}B_{M}^{*}, & \text { if } M=N, \\
B_{M}^{*}+\lambda_{M N}, & \text { if } M \supset N, \\
B_{M}^{*}-\lambda_{M N}, & \text { if } M \subset N .\end{cases}\right.
$$

We see that if $M_{1} \supset M_{2}$, then $\left.A_{M_{1}}^{\prime}\right|_{M_{2}}=A_{M_{2}}^{\prime}$ and $\left.\left(B_{M_{2}}^{*}\right)^{\prime}\right|_{\left(\left(M_{1}\right)_{-}\right)^{\perp}}=\left(B_{M_{1}}^{*}\right)^{\prime}$. Thus we can define a linear mapping $A: \bigcup\left\{N: N \in \mathscr{N}, N_{-} \neq X\right\} \rightarrow X$, such that $\left.A\right|_{M}=A_{M}^{\prime}$ whenever $M \in \mathscr{N}$ and $M_{-} \neq X$. Similarly, we can define a linear mapping $B^{*}: \bigcup\left\{\left(N_{-}\right)^{\perp}: N \in \mathscr{N}, N_{-} \neq 0\right\} \rightarrow X^{*}$, such that $\left.B^{*}\right|_{\left(N_{-}\right)^{\perp}}=\left(B_{N}^{*}\right)^{\prime}$ whenever $N \in \mathcal{N}$ and $N \neq 0$. Therefore the mappings $A, B$ satisfy the requirement of the lemma.

Lemma 3.4. If $\phi$ is norm-continuous, then the mappings $A, B^{*}$ in Lemma 3.3 are bounded on their domains. Therefore $A$ can be extended to a bounded linear operator $A$ on all of $X$. Likewise, $B^{*}$ can be extended to a bounded linear operator on all of $X^{*}$.

Proof. Fix $N \in \mathscr{N}$ such that $N_{-} \neq X$ and $N \neq 0$. Because $\phi$ is bounded, it follows from the closed graph theorem that $\left.A\right|_{N}$ and $\left.B^{*}\right|_{\left(N_{-}\right)^{\perp}}$ are bounded. Now let $0 \neq M \in \mathscr{N}$, and let $f \in\left(M_{-}\right)^{\perp}$, with $\|f\|=1$. If $M \supseteq N$, then we have $f \in\left(N_{-}\right)^{\perp}$, and hence $\left\|B^{*} f\right\| \leq\left\|\left.B^{*}\right|_{\left(N_{-}\right)^{\perp}}\right\|$. On the other hand, suppose that $M \subset N$ and choose any $x_{0} \in M$ with $\left\|x_{0}\right\|=1$; then $\left\|B^{*} f\right\|=$ $\left\|x_{0} \otimes B^{*} f\right\|=\left\|\phi\left(x_{0} \otimes f\right)-A x_{0} \otimes f\right\|=\left\|\phi\left(x_{0} \otimes f\right)-\left.A\right|_{N} x_{0} \otimes f\right\| \leq\|\phi\|+\left\|\left.A\right|_{N}\right\|$. Because $\mathscr{N}$ is a nest and $M$ is arbitrary, we see that $B^{*}$ is bounded on its domain.

To show that $A$ is bounded on its domain, let $M \in \mathscr{N}$ be such that $0 \neq M_{-}$ and $M_{-} \neq X$. Choose $x \in M$ with $\|x\|=1$. Now pick $f_{0} \in\left(M_{-}\right)^{\perp}$ with $\left\|f_{0}\right\|=1$. Then we have $\|A x\|=\left\|A x \otimes f_{0}\right\|=\left\|\phi\left(x \otimes f_{0}\right)-x \otimes B^{*} f_{0}\right\| \leq$ $\|\phi\|+\left\|B^{*}\right\|$. Thus $A$ is bounded on its domain.

Corollary 3.5. If $X$ is a reflexive Banach space, then there exist $A, B \in B(X)$ such that $\phi(x \otimes f)=A x \otimes f+x \otimes B$ for all $x \otimes f \in \operatorname{Alg} \mathscr{N}$.

Proof. Let $B=\left(B^{*}\right)^{*}$. Then the conclusion follows from Lemma 3.4. 
Corollary 3.6. If $X$ is a reflexive Banach space and $\phi$ is continuous in the strong operator topology, then $\phi \in \mathscr{D}(\mathcal{N})$.

Proof. By Lemma 2.2 and Corollary 3.5, there exist $A, B \in B(X)$ such that $\phi(T)=A T+T B$ for all $T \in \operatorname{Alg} \mathscr{N}$. By the remark following Lemma 2.4, every member of $\mathscr{D}(\mathcal{N})$ is inner, and therefore, because $\phi$ is a local derivation, we must have $\phi(I)=0$, i.e., $B=-A$. Thus, $\phi(T)=A T-T A$ for all $T \in \operatorname{Alg} \mathscr{N}$. Hence $\phi$ is in fact a derivation.

Theorem 3.7. Suppose that $X$ is reflexive and $\phi$ is norm-continuous. Moreover, suppose that $\mathcal{N}$ satisfies the following condition: For all $0 \subset M \in \mathscr{N}$ and for all $X \supset N \in \mathcal{N}$, there exist $E_{1} \in(0, M]$ and $E_{2} \in(N, X]$ such that $\left(E_{1}\right)_{-} \subset E_{1}$ and $\left(E_{2}\right)_{-} \subset E_{2}$. Then $\phi \in \mathscr{D}(\mathscr{N})$.

Proof. Let $A, B \in B(X)$ be as in Corollary 3.5, and for $T \in \operatorname{Alg} \mathcal{N}$ define $\phi_{0}(T)=\phi(T)-(A T+T B)$. Then $\phi_{0}$ is a linear mapping from $\operatorname{Alg} \mathscr{N}$ into $B(X)$ such that $\phi_{0}(F)=0$ for all finite rank operators $F \in \operatorname{Alg} \mathscr{N}$. We claim that $\phi_{0}=0$. Otherwise, there exist $T \in \operatorname{Alg} \mathscr{N}$ and $x \in X$ such that $\phi_{0}(T) x \neq 0$. By hypothesis, we may assume that there exists $0 \neq E_{1} \in \mathscr{N}$ such that $\left(E_{1}\right)_{-} \subset E_{1}$ and $\phi_{0}(x) \in\left(E_{1}\right)_{-}$. Likewise, by hypothesis, we may assume that there exists $E_{2} \in \mathcal{N}$ such that $\left(E_{2}\right)_{-} \subset E_{2}$, with $x \in E_{2}$ and $x \notin\left(E_{2}\right)_{-}$. Choose $f \in\left(E_{2}\right)_{-}^{\perp}, g \in\left(E_{1}\right)_{\perp}^{\perp}, y \in E_{1}$ such that $f(x)=1, g\left(\phi_{0}(T) x\right)=1$, $g(y)=1$. Then $x \otimes f$ and $y \otimes g$ are projections in $\operatorname{Alg} \mathscr{N}$. Therefore, $y \otimes g \phi_{0}((I-y \otimes g) T(I-x \otimes f)) x \otimes f=0$. Because $T-(I-y \otimes g) T(I-x \otimes f)$ is of finite rank, we have that $\phi_{0}(T-(I-y \otimes g) T(I-x \otimes f))=0$. It follows that $0=(y \otimes g) \phi_{0}(T)(x \otimes f)=g\left(\phi_{0}(T) x\right) y \otimes f$. Thus $g\left(\phi_{0}(T) x\right)=0$, which produces a contradiction. Therefore, $\phi(T)=A T+T B$ for all $T \in \operatorname{Alg} \mathscr{N}$. As in the proof of Corollary 3.6, $\phi(I)=0$; hence $B=-A$, i.e., $\phi(T)=A T-T A$ for all $T \in \operatorname{Alg} \mathscr{N}$. Hence $\phi$ is a derivation.

Corollary 3.8. Let $X$ be as in Theorem 3.7. Then $\mathscr{D}(\mathcal{N})$ is (topologically) algebraically reflexive.

Corollary 3.9. If $\operatorname{dim} X<\infty$, then $\mathscr{D}(\mathscr{N})$ is algebraically reflexive.

Corollary 3.10. Let $X$ be reflexive. Suppose that $\mathscr{N}$ has the property that $0_{+} \neq$ 0 and $X_{-} \neq X$. Then $\mathscr{D}(\mathscr{N})$ is (topologically) algebraically reflexive.

\section{ACKNOWLEDGMENT}

We wish to express our thanks to David Larson and Randy Crist for their useful comments on this paper.

\section{REFERENCES}

[ALVS] A. Loginov and V. Sulman, Hereditary and intermediate reflexivity of $\mathbf{W}^{*}$-algebras, Izv. Akad. Nauk USSR 39 (1975), 1260-1273; English transl., Math. USSR-Izv. 9 (1975), 1189-1201.

[DH] D. Hadwin, Algebraically reflexive linear transformations, Linear and Multilinear Algebra 14 (1983), 225-233.

[H1] Han Deguang, Derivations and isomorphisms of certain reflexive operator algebras, submitted. 
[H2] - Rank one operators and bimodules of reflexive operator algebras in Banach spaces, J. Math. Anal. Appl. 161 (1991), 188-193.

[H3] Cohomology of certain reflexive operator algebras, submitted.

[H4] - The first cohomology groups of nest algebras on normed spaces, Proc. Amer. Math. Soc. 118 (1993), 1147-1149.

[K] R. V. Kadison, Local derivations, J. Algebra 130 (1990), 494-509.

[L] D. R. Larson, Reflexivity, algebraic reflexivity and linear interpolation, Amer. J. Math. 110 (1988), 283-291.

[LS] D. R. Larson and A. R. Sourour, Local derivations and local automorphisms of $B(X)$, Proc. Sympos. Pure Math., vol. 51, Amer. Math. Soc., Providence, RI, 1992, pp. 187-192.

[Lam] M. S. Lambrou, Approximants, commutants and double commutants in normed algebras, $\mathrm{J}$. London Math. Soc. (2) 25 (1982), 499-512.

[Spa] N. K. Spanoudakis, Generalizations of certain nest algebra results, Proc. Amer. Math. Soc. 115 (1992), 711-723.

Department of Mathematics, Qufu Normal University, Qufu, Shandong, 273165 PeoPLE'S REPUBLIC OF CHINA

E-mail address: deguang.han@math.tamu.edu 